\title{
EVIDÊNCIA DE VALIDADE DE CONSTRUTO CONVERGENTE PARA O TESTE DE ATENÇÃO DIVIDIDA - TEADI
}

\author{
Fabián Javier Marín Rueda \\ Psicólogo e Doutor em Avaliação Psicológica pela Universidade São Francisco. Docente do Programa \\ de Pós-graduação Stricto Sensu em Psicologia da Universidade São Francisco/Itatiba. \\ Nelimar Ribeiro de Castro \\ Psicólogo, Mestre em Avaliação Psicológica e Doutorando do Programa de Pós-graduação Stricto \\ Sensu em Psicologia da Universidade São Francisco/Itatiba.
}

\begin{abstract}
Resumo
O objetivo do estudo foi buscar evidências de validade de construto convergente para o Teste de Atenção Dividida (TEADI) por meio da correlação com os Testes de Atenção Dividida e Sustentada (AD e AS). Participaram da pesquisa 105 estudantes universitários de uma instituição particular do estado de Sergipe, com idade variando de 18 a 53 anos $(M=24,95, D P=6,62)$, sendo $41,9 \%$ do sexo masculino. Os instrumentos foram aplicados de forma coletiva, com duração aproximada de 30 minutos. Os resultados evidenciaram correlações de magnitudes moderadas e altas entre o TEADI e as medidas do AD, e baixas e nulas entre o TEADI e as medidas do AS. Esses dados eram esperados, uma vez que o TEADI e o AD se propõem a avaliar o mesmo tipo de atenção, enquanto o AS sugere avaliar um tipo diferente, qual seja, a atenção sustentada.
\end{abstract}

Palavras-chave: atenção, psicometria, evidências de validade, avaliação psicológica.

\section{EVIDENCE OF CONVERGENT CONSTRUCT VALIDITY OF THE DIVIDED ATTENTION TEST - TEADI}

\begin{abstract}
The objective of the study was to search for evidences of convergent construct validity for the Test of Divided Attention (TEADI) by the correlation with the Tests of Divided Attention and Sustained Attention (AD and AS). 105 college students of a private institution of the state of Sergipe had participated of the research, from 18 to 53 years $(M=24.95, S D=6.62), 41.9 \%$ male. The instruments had been applied collectively, with approach duration of 30 minutes. The results had evidenced correlations from moderate to high magnitudes between the TEADI and the measures of the AD, and low and null magnitudes between the TEADI and the measures of the AS. These data were expected, since the TEADI and the AD are proposed to evaluate the same type of attention, whereas the AS suggests evaluating a different type, the sustained attention.

Keywords: attention, psychometry, validity evidences, psychological assessment.
\end{abstract}

\section{EVIDENCIA DE VALIDEZ DE CONSTRUCTO CONVERGENTE PARA EL TEST DE ATENCIÓN DIVIDIDA - TEADI}

\begin{abstract}
Resumen
El objetivo del estudio fue buscar evidencias de validez de constructo convergente para el Test de Atención Dividida (TEADI) por medio de la correlación con los Testes de Atención Dividida y Sostenida ( $A D$ y $A S$ ). Participaron de la investigación 105 estudiantes universitarios de una institución privada del estado de Sergipe, con edad
\end{abstract}


variando de 18 a 53 años $(P=24,95, D T=6,62)$, siendo $41,9 \%$ del sexo masculino. Los instrumentos fueron aplicados de forma colectiva con una duración aproximada de 30 minutos. Los resultados mostraron correlaciones de magnitudes moderadas y altas entre el TEADI y las medidas del AD, y bajas y nulas entre el TEADI y las medidas del AS. Esos datos eran esperados, pues el TEADI y el AD tienen como objetivo evaluar el mismo tipo de atención, mientras que el AS sugiere evaluar un tipo de atención diferente, que sería la atención sostenida.

Palabras clave: atención, psicometría, evidencias de validez, evaluación psicológica.

\section{INTRODUÇÃO}

A atenção pode ser definida como o processo mental que administra a grande quantidade de informações disponibilizadas ao organismo por meio dos sentidos, da memória e de outros processos cognitivos, permitindo a seleção dos estímulos julgados relevantes, em detrimento daqueles avaliados como irrelevantes (Gazzaniga, Ivry \& Mangun, 2006; Sternberg, 2000). Nesse sentido, James em 1890 apresentou a idéia de que o indivíduo exerceria o controle ativo de suas experiências focalizando sua atenção naquilo que escolhesse ou nos estímulos que por si só atrairiam sua atenção. Para o autor, três funções básicas estariam presentes na atenção, quais sejam, a capacidade de seu controle voluntário, a capacidade limitada do processamento da informação e a inabilidade de atender diversos estímulos simultaneamente expressa no caráter seletivo e de focalização (Braga, 2007; Gazzaniga et al, 2006; Lima, 2005).

A focalização da atenção, também chamada de atenção focalizada ou atenção seletiva é o tipo mais elementar de atenção, sendo geralmente definida como a capacidade de selecionar um estímulo dentre vários outros (Benczik \& Casella, 2007; Braga, 2007). Normalmente operacionaliza-se o estudo desse tipo de atenção apresentando-se às pessoas vários estímulos e solicitando que respondam a apenas um deles (Braga, 2007). Vale destacar que essa operacionalização da atenção, no Brasil, é comumente classificada como atenção concentrada (Cambraia, 2003; Rueda \& Sisto, 2009).

Porém, o próprio Willian James já admitia a possibilidade de dois estímulos serem atendidos simultaneamente caso um dos processos ocorresse automaticamente (James, 1890, citado por Braga, 2007). Um processo automático não envolveria o controle consciente e exigiria pouco ou nenhum esforço para ser realizado. Em oposição, um processo voluntário ou controlado, não somente se mostraria acessível ao controle consciente como também o exigiria (Sternberg, 2000). 
Sternberg (2000) relatou uma série de experimentos datados do início do estudo sobre a atenção pela psicologia cognitiva (década de 1960 do século XX) envolvendo duas tarefas simultâneas. Os estudos mostraram a possibilidade de duas tarefas serem atendidas simultaneamente, contudo, alguns aspectos podem favorecer ou dificultar o desempenho nas tarefas duplas. Inicialmente, a execução de duas tarefas conscientes reduz o desempenho em ambas, entretanto, se uma das tarefas for automatizada, por exemplo, pelo treino, ocorreria a melhora no desempenho em ambas as tarefas. Além disso, tarefas duplas envolvendo modalidades sensoriais diferentes seriam mais fáceis de serem realizadas.

Esse tipo de distribuição atencional ficou conhecido como atenção dividida. $\mathrm{Na}$ atenção dividida ocorreria a distribuição dos recursos atencionais a mais de um estímulo, estando relacionada à possibilidade de responder a mais de um estímulo ou efetuar mais de uma ação simultaneamente (Benczik \& Casella, 2007; Gaddes \& Edgell, 1994; Sternberg, 2000). Avalia-se a atenção dividida solicitando que a pessoa focalize pelo menos dois estímulos enquanto é exposta a estímulos concorrentes, que exercem a função de distratores, distribuídos entre os estímulos-alvo (Sisto, Noronha, Lamounier, Bartholomeu \& Rueda, 2006).

A atenção sustentada tem sido outro tipo de atenção bastante investigado. Por atenção sustentada entende-se a capacidade de manutenção do foco atencional por um longo período de tempo durante uma atividade repetitiva ou continua (Benczik \& Casella, 2007; Gaddes \& Edgell, 1994). Assim, essa tarefa demanda alta concentração e velocidade de processamento quando é imposto tempo de execução (Sisto \& cols., 2006).

No que se refere à atenção concentrada, ela é outro tipo também bastante avaliado. Defini-se atenção concentrada como a capacidade de selecionar um estímulo ou grupo de estímulos diante de muitos outros e manter sua atenção para esse estímulo por um longo período de tempo a fim de executar com qualidade uma tarefa especifica (Cambraia, 2003).

Os conceitos de atenção sustentada e a concentrada são freqüentemente confundidos e considerados como o mesmo tipo de atenção. Contudo, existem algumas diferenças entre elas que as distinguem. $\mathrm{Na}$ atenção sustentada avaliase a sustentação criando condições para que a pessoa chegue até o final do teste diferenciando-se da avaliação da atenção concentrada para a qual se estabelece 
um tempo limite de execução da tarefa independentemente se a pessoa chegará ou não até o final do teste enquanto ela focaliza um estímulo (Baptista, Rueda \& Sisto, 2007).

Noronha, Sisto, Bartholomeu e Rueda (2006) realizaram um estudo comparando esses dois tipos de atenção conforme avaliados pelo Teste de Atenção Sustentada (AS) e o Teste de Atenção Concentrada (AC). Foram encontradas correlações significativas, porém baixas (entre 0,27 e 0,40), sendo um pouco menores quando controlado o efeito da idade (ficando entre 0,22 e $0,35)$. Os autores concluíram que os construtos avaliados pelos dois testes possuíam certa comunalidade, indicando que avaliavam construtos semelhantes, mas não iguais.

Tais inferências baseiam-se no próprio significado da correlação entre duas medidas. $O$ índice de correlação pode variar entre -1 e +1 , sendo que, os valores negativos indicam relações inversas e os positivos sugerem associações diretas entre as variáveis. Além disso, correlações iguais a um representam associações perfeitas, enquanto que iguais a zero indicam nenhuma relação (Sisto, 2007). Em psicometria, os índices de correlação têm sido utilizados como evidências de validade de construto (Pasquali, 2001).

Por validade de um teste entende-se aquilo que ele mede e o quão bem ele o faz. Especificamente, entende-se por validade de construto a extensão em que se pode dizer que um teste mede um determinado construto teórico (Anastasi \& Urbina, 2000). Assim, ao utilizar a correlação entre um novo teste com testes semelhantes já existentes, buscam-se evidências de que eles mensuram a mesma área de comportamento, esperando-se, desse modo, correlações moderadamente altas (Arías, 1996; Anastasi \& Urbina, 2000; Pasquali, 2001). A hipótese testada nesse tipo de validade é que "se o teste $X$ mede validamente 0 traço $Z$, e o novo teste $\mathrm{N}$ se correlaciona altamente com o teste $\mathrm{X}$, então o novo teste mede o mesmo traço medido por aquele teste" (Pasquali, 2001, p. 120), justamente porque, quanto mais próximo a 1 (um) for o índice de correlação, maior a comunalidade entre duas variáveis (Sisto, 2007).

Desse modo, o resultado relatado na pesquisa de Noronha et al (2006), ao associar o AC e o AS, indicou evidências de validade do tipo convergente entre esses testes. Com o mesmo objetivo outros autores investigaram a relação entre outros testes de atenção. Sisto et al (2006), por exemplo, correlacionaram o Teste de Atenção Dividida (AD) com o Teste de Atenção Concentrada ( $A C$ ). 
Nesse estudo participaram 210 sujeitos com idade entre 18 e 62 anos e de ambos os sexos. O AC foi correlacionado com duas medidas oferecidas pelo $A D$, quais sejam, Velocidade com Qualidade e Concentração. Para a primeira os índices de correlação variaram entre 0,39 e 0,56, e para a segunda entre 0,47 e 0,49 , sendo, desse modo, todos de magnitude moderada e significativas. Quando foi controlado o efeito da idade as correlações mantiveram-se nos mesmos níveis.

O AD e o AS também foram associados ao Teste de Atenção Concentrada (TEACO-FF). Com o AD no índice Velocidade com Qualidade as correlações foram nulas para a amostra total sem e com o controle da idade $(r=0,14$ e $r=0,19$ respectivamente), e para as mulheres, sendo 0,11 e 0,15 respectivamente. Contudo, para os homens a correlação foi baixa tanto para a correlação parcial com controle do efeito da idade quanto sem esse controle $(r=0,29$ e $r=0,19$ respectivamente). Já com o índice de Concentração as correlações foram baixas para a amostra total $(r=0,39$ e $r=0,37)$ e mulheres $(r=0,35$ e $r=0,29)$ e altas para os homens $(r=0,73$ e $r=0,75)$ sem e com o controle do efeito da idade. Sendo que apenas o índice de Concentração obteve correlações significativas (Rueda, 2009).

Por sua vez, com o AS, nos índices de Concentração e Velocidade com Qualidade, as correlações por sexo e na amostra total foram moderadas, entre 0,61 e 0,71, ficando muito próximos a esses valores, 0,59 e 0,69, quando se controlou o efeito da idade. O autor concluiu que o TEACO-FF e o AS apresentam similaridade na forma de operacionalização do construto, pois no AS o sujeito deve localizar apenas um estímulo dentre vários distratores, o que seria característico da atenção concentrada (Rueda, 2009).

Por sua vez, Rabelo et al (2009) relacionaram o TEACO-FF e o Teste das Trilhas Coloridas - TTC (Color Trails Test - CTT) com o objetivo de buscar evidências de validade para o TTC. Esse teste mede a atenção sustentada, em sua Forma 1, e a atenção dividida e alternada em sua Forma 2. Participaram da pesquisa 21 pessoas entre 41 e 79 anos, de ambos os sexos. Foram encontrados índices de correlação positivos e moderados entre os testes. Também em um estudo de evidência de validade, Castro, Sisto e Silva (2009) associaram o Teste de Atenção Seletiva Visual e o teste Medida e Prontidão Mental (MPM). A amostra foi constituída por 174 participantes com idade entre 18 e 54 anos, de ambos os sexos e diversos níveis de escolaridade. As correlações encontradas entre os 
testes foram positivas e moderadas, indicando evidência de validade para o Teste de Atenção Seletiva Visual.

Em contraposição a esses resultados, a correlação entre testes que sugerem avaliar o mesmo tipo de atenção apresentaram índices mais elevados. Nesse sentido, encontram-se estudos com o AC, que obtiveram correlações positivas de magnitudes moderadas a muito altas quando associado com outros testes de atenção concentrada, tais como o Teste de Atenção Concentrada de ToulousePiéron $(r=0,93)$, o TACOM-A $(r=0,63)$, o TACOM-B $(r=0,62)$ e o Teste D2 $(r=0,42)$, sendo todas significativas (Cambraia, 2003).

O AC também foi utilizado para buscar evidência de validade para o Teste de Atenção Concentrada (TEACO-FF). Do estudo participaram 340 pessoas, com idades entre 18 e 59 anos e de ambos os sexos. A correlação entre os testes encontrou índices moderados tanto para os homens $(r=0,52)$ quanto para as mulheres $(r=0,51)$ e amostra total $(r=0,51)$. Com pequena redução para mulheres $(r=0,46)$ e total $(r=0,49)$ quando foi controlado o efeito da idade (Rueda, 2009). As diferenças de correlação encontradas indicaram que o AC possui maior comunalidade com o Teste de Atenção Concentrada de ToulousePiéron, seguido pelo TACOM-A e B, e menor com o D2 e o TEACO-FF (Cambraia, 2003; Rueda, 2009).

Em suma, os estudos de validade entre testes de atenção têm encontrado correlações moderadas e altas entre instrumentos que avaliam o mesmo tipo de atenção, e baixas e moderadas entre aqueles que mensuram tipos diferentes, indicando maior comunalidade no primeiro caso (Cambraia, 2003; Castro et al, 2009; Noronha et al, 2006; Rabelo et al, 2009; Rueda, 2009; Sisto et al, 2006). Nesse sentido, este estudo tem por objetivo buscar evidências de validade para o Teste de Atenção Dividida - TEADI (Rueda, 2010) por meio da correlação com os Testes de Atenção Dividida e Sustentada (AD e AS) (Sisto et al, 2006).

\section{MÉTODO}

\section{Participantes}

Participaram da pesquisa 105 estudantes de uma universidade particular do estado de Sergipe, que estavam matriculados em diversos cursos. Em relação à idade, ela variou de 18 a 53 anos $(M=24,95, D P=6,62)$. Quanto ao sexo, 44 $(41,9 \%)$ eram homens e $61(58,1 \%)$ mulheres. 


\section{Instrumentos}

Teste de Atenção Dividida - TEADI (Rueda, 2010)

O TEADI fornece uma medida referente à capacidade da pessoa dividir a atenção. $O$ instrumento possui 450 estímulos distribuídos em 30 linhas com 15 estímulos cada. O resultado obtido no TEADI pode ser calculado com base nos estímulos que a pessoa deveria marcar e marcou, subtraído dos erros (estímulos que não deveriam ser marcados e foram) e das omissões (estímulos alvo que não foram marcados pela pessoa). $O$ tempo de aplicação é de 5 minutos.

Em relação às propriedades psicométricas, um estudo inicial foi realizado por Rueda (2009) com o objetivo de verificar o desempenho do teste em função da idade das pessoas. Dessa forma, os resultados mostraram correlação negativa e significativa entre a pontuação do TEADI e a idade das pessoas. Posteriormente, a análise de variância mostrou diferenças significativas entre as idades e, com base nisso, foram formadas três faixas etárias que se diferenciaram pela prova de Tukey. Tais faixas etárias foram, até os 25 anos, de 26 a 35 anos e a faixa etária de 36 anos ou mais. Com base nisso, o autor concluiu por evidência de validade para o TEADI com base na diferenciação das idades. No que tange aos índices de precisão, eles foram calculados pelo alfa de Cronbach e pelo método das duas metades de Guttman e Spearman-Brown, para cada uma das faixas etárias e para a amostra geral. Os resultados evidenciaram uma variação entre 0,83 e 0,89, sendo eles considerados excelentes. Dessa forma, concluiu-se que a quantidade de erro de medida presente no instrumento era baixa.

Testes de Atenção Dividida e Sustentada - AD e AS (Sisto, Noronha, Lamounier, Bartholomeu \& Rueda, 2006)

O $A D$ foi construído tendo como amostra sujeitos que passaram pelo processo de avaliação psicológica pericial para obtenção da CNH e fornece dois tipos de informação, quais sejam, a Concentração e a Velocidade com Qualidade. A Concentração é calculada somando os itens que eram para serem assinalados menos erros e omissões, enquanto a medida da Velocidade com Qualidade calcula-se somando a quantidade de itens que eram para marcar e o indivíduo marcou (tarefa solicitada) mais os itens que não eram para serem marcados e não o foram, menos os erros e as omissões. O tempo total para a realização da tarefa é de 5 minutos. 
Nas propriedades psicométricas do teste foi verificada evidência de validade desenvolvimental e com o Psicodiagnóstico Miocinético - PMK (Mira, 2004). A precisão do instrumento foi calculada pelo Alfa de Cronbach, obtendo índices que variaram de 0,87 a 0,97, considerados excelentes pelos autores.

O AS também foi desenvolvido e normatizado para candidatos à obtenção da $\mathrm{CNH}$. Ele avalia a capacidade da pessoa manter o foco em um aspecto por um longo período, ao mesmo tempo em que estão presentes elementos distratores, tais como estímulos que não são pertinentes ou baixa freqüência do estímulo que não se pode perder de vista, sendo imposto um tempo de execução igual para o começo e o final do teste.

Do instrumento são extraídas três informações, a saber, a Concentração, a Velocidade com Qualidade e a Sustentação. A Concentração corresponde à soma de itens que eram para serem assinalados (tarefa solicitada) menos erros e omissões. A Velocidade com Qualidade diz respeito à quantidade de itens que o indivíduo realizou ao todo (itens que eram para serem marcados e foram mais os itens que não eram para serem assinalados e não o foram) menos os erros e omissões. Para classificar o nível de Sustentação da pessoa são somados os itens que eram para marcar e foram marcados (solicitação da tarefa) com os itens que não eram para serem marcados e não o foram das três primeiras linhas. Desse total, a soma dos erros com as omissões é subtraída.

Esse mesmo procedimento é repetido com as três últimas linhas. Obtidos esses dois índices, o segundo é subtraído do primeiro e o resultado é interpretado conforme as tabelas de normatização do Manual, sendo verificado posteriormente se a pessoa manteve, perdeu ou ganhou Sustentação. Para responder o teste a pessoa possui 15 segundos para responder cada fileira de estímulos. Dessa forma, o tempo total é de 6'15".

Quanto às propriedades psicométricas do teste, num primeiro momento foi estudada a estrutura interna, sendo que a análise fatorial forneceu dois fatores explicando $70,09 \%$ da variância. Como no primeiro fator a carga fatorial foi bem maior e a relação entre os eigenvalues do primeiro e segundo fator foi maior que 14 , os autores concluíram que os subtestes podiam ser explicados por apenas um fator, ou seja, que o AS possuiria uma única dimensão. Ainda, evidência de validade de construto desenvolvimental foi verificada por meio do estudo das diferentes faixas etárias. Também, evidência de validade divergente foi verificada quando comparado com o Psicodiagnóstico Miocinético - PMK (Mira, 2004). 
Em relação à precisão, ela foi estudada pelo alfa de Cronbach, o coeficiente de consistência interna e o método das metades, sendo que por este último foram fornecidos os resultados pelos procedimentos de Guttman e SpearmanBrown. Os coeficientes variaram de 0,73 até 0,97. Por esses dados os autores concluíram que o teste apresentou bons índices de precisão.

\section{Procedimentos}

Num primeiro momento estabeleceu-se contato com a instituição de ensino superior de Sergipe explicando os propósitos da pesquisa, sendo posteriormente a mesma encaminhada ao Comitê de Ética da Universidade São Francisco. Após a aprovação procedeu-se à coleta de dados, com a assinatura do Termo de Consentimento Livre e Esclarecido por parte dos indivíduos participantes da pesquisa.

A aplicação dos testes ocorreu de forma coletiva em horário previamente combinado com o respectivo professor. Para isso foram treinados dois estagiários do curso de psicologia que deram as instruções em turmas de, no máximo, 30 alunos.

A ordem de aplicação foi a mesma em todas as turmas. Em primeiro lugar foi aplicado o TEADI, seguido do AD e, por fim, o AS. O tempo total da coleta não excedeu os 25 minutos.

\section{RESULTADOS}

$\mathrm{Na}$ análise dos resultados foram calculadas as estatísticas descritivas dos três instrumentos e suas respectivas medidas, posteriormente realizou-se uma correlação da pontuação do TEADI com as medidas de Concentração e de Velocidade com Qualidade do AD e do AS, sendo que na medida de Sustentação do AS realizou-se uma análise de variância. Por fim, levando em consideração que os três testes apresentam evidência de validade relativa à idade das pessoas, optou-se por realizar uma correlação parcial controlando o efeito dessa variável na relação entre os testes.

Assim, as estatísticas descritivas referentes ao TEADI mostraram uma pontuação média de 120,78 pontos ( $(D P=43,11)$. A Figura 1 é apresentada para elucidar essas informações. 


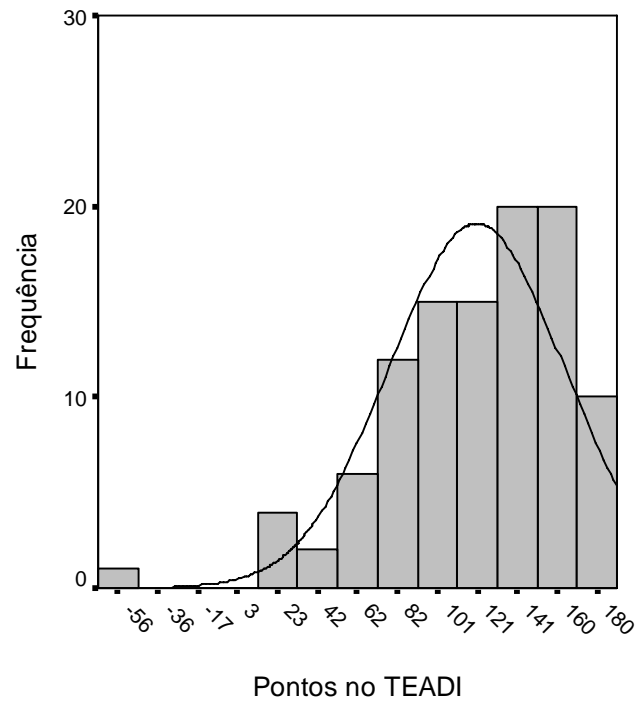

Figura 1.

Freqüência de pontos dos participantes no TEADI.

Ainda pode ser verificado que a moda foi 110 e a mediana 128, tendo havido uma concentração de pontos entre 125 e 165 pontos (44,9\%). Apenas uma pessoa apresentou pontuação negativa, o que indicou que a mesma errou e omitiu mais estímulos do que acertou.

Quanto às informações das medidas fornecidas pelo $A D$, pode ser observada a Figura 2. Em relação a elas, pode-se dizer que a média de pontos em Concentração foi de 34,30 pontos ( $D P=28,59)$ e em Velocidade com Qualidade de $121,74(\mathrm{DP}=44,59)$.
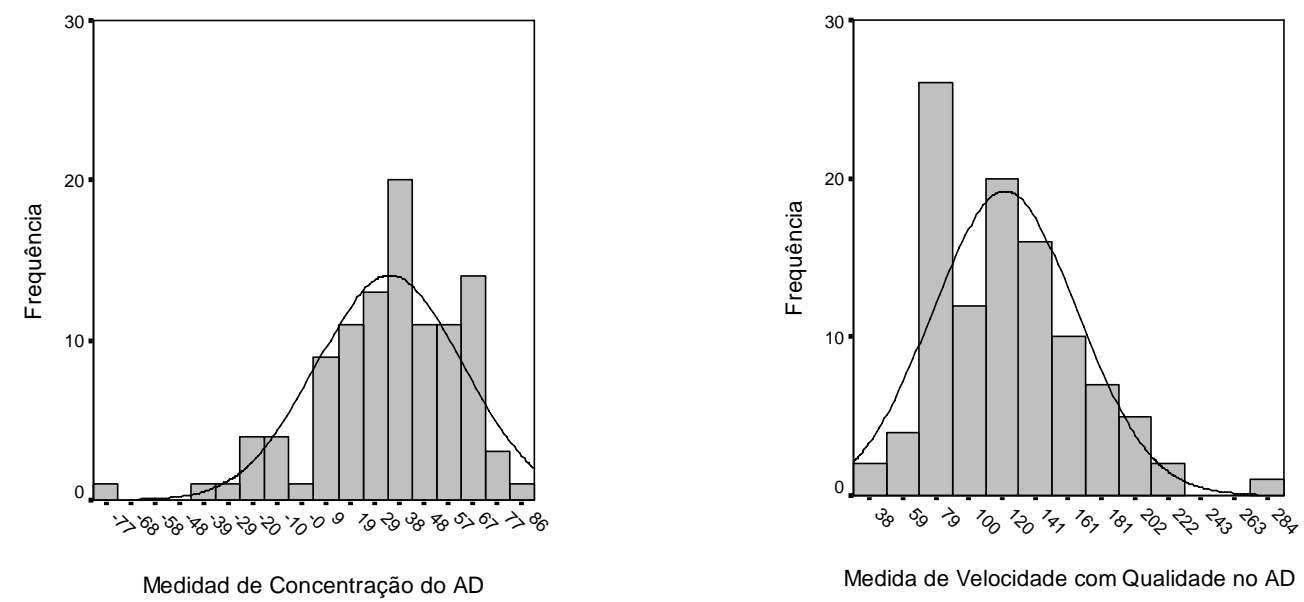

Figura 2.

Freqüência de pontos dos participantes nas medidas do AD. 
Já a moda foi de 42 em Concentração e de 73 em Velocidade com Qualidade. Por sua vez, a mediana foi de 37 e 120 respectivamente. Em relação às pontuações negativas, foram verificadas apenas na medida de Concentração, na qual houve $11(10,5 \%)$ pessoas que acertaram menos do que erraram e omitiram.

Por fim, a Figura 3 se refere às medidas de Concentração e de Velocidade com Qualidade do AS. A média de pontos foi 51,13 em Concentração e 119,98 em Velocidade com Qualidade ( $D P=17,34$ e $D P=24,48$ respectivamente).
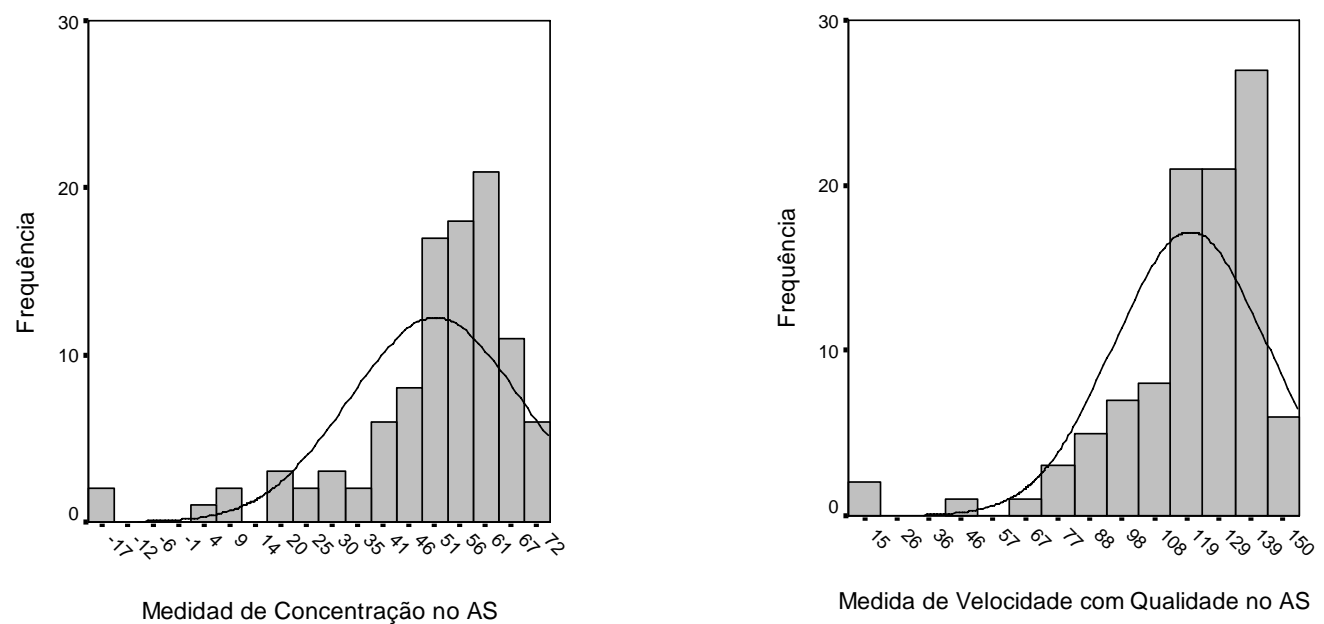

Figura 3.

Freqüência de pontos dos participantes nas medidas do AS.

Nas pontuações, apenas duas pessoas tiveram pontuações negativas (ambas na medida de Concentração). A moda em Concentração foi de 58 pontos e a mediana de 55. Em Velocidade com Qualidade a moda e a mediana foram de 124 pontos.

Quanto à medida de Sustentação fornecida pelo teste, destaca-se que 16 pessoas $(15,7 \%)$ diminuíram a sua sustentação, enquanto que 7 indivíduos $(6,9 \%)$ tiveram um aumento. Já a quantidade de sujeitos que manteve o nível de Sustentação representou 77,5\% (79 pessoas).

Para verificar a relação entre os instrumentos foi realizada uma correlação de Pearson na amostra geral e em cada uma das faixas etárias diferenciadas pelo TEADI. Os resultados podem ser observados na Tabela 1. 
Tabela 1.

Coeficientes de Correlação de Pearson entre o TEADI e as medidas de Concentração e de Velocidade com Qualidade do AD e AS, no total e por faixa etária.

\begin{tabular}{|c|c|c|c|c|c|}
\hline & & \multicolumn{4}{|c|}{$A D$} \\
\hline & & \multicolumn{2}{|c|}{ Concentração } & \multicolumn{2}{|c|}{$\begin{array}{c}\text { Velocidade com } \\
\text { Qualidade }\end{array}$} \\
\hline & & $r$ & $p$ & $r$ & $p$ \\
\hline \multirow[t]{7}{*}{ TEADI } & Até 25 anos & 0,75 & $<0,001$ & 0,65 & $<0,001$ \\
\hline & 26 a 35 anos & 0,77 & $<0,001$ & 0,44 & 0,044 \\
\hline & 36 anos ou & 0,57 & 0,034 & 0,51 & 0,036 \\
\hline & Amostra total & 0,74 & $<0,001$ & 0,66 & $<0,001$ \\
\hline & & \multicolumn{4}{|c|}{ AS } \\
\hline & & \multicolumn{2}{|c|}{ Concentração } & \multicolumn{2}{|c|}{$\begin{array}{c}\text { Velocidade com } \\
\text { Qualidade }\end{array}$} \\
\hline & & $r$ & $p$ & $r$ & $p$ \\
\hline \multirow[t]{4}{*}{ TEADI } & Até 25 anos & 0,25 & 0,033 & 0,29 & 0,015 \\
\hline & 26 a 35 anos & 0,09 & 0,652 & 0,19 & 0,362 \\
\hline & 36 anos ou & 0,31 & 0,613 & 0,42 & 0,482 \\
\hline & Amostra total & 0,29 & 0,003 & 0,35 & $<0,001$ \\
\hline
\end{tabular}

Pela Tabela 1 pode ser observado que todas as correlações entre o TEADI e o AD foram positivas e estatisticamente significativas, variando entre magnitudes altas e moderadas. No caso da correlação com a medida de Concentração desse instrumento e o TEADI, as correlações foram de magnitude alta, exceção feita à faixa etária de 36 anos ou mais, que apresentou uma correlação moderada, de 0,57. Quanto à amostra total, a correlação foi de 0,74 , evidenciando uma comunalidade de quase $55 \%$ entre a atenção dividida avaliada pelo TEADI e a atenção dividida avaliada pela medida de Concentração do AD. Isso evidencia que ambos os instrumentos avaliam grande parte do mesmo construto.

Já falando da relação entre a medida de Velocidade com Qualidade do AD com o TEADI, a magnitude da correlação para a faixa etária até os 25 anos e para a amostra total foi alta, enquanto que para as faixas etárias de 26 a 35 anos e de 36 anos ou mais, a magnitude das correlações foi moderada. Quanto à correlação das medidas na amostra total a comunalidade foi de quase $45 \%$.

Em relação à Concentração e Velocidade com Qualidade do AS, as análises mostraram que apenas na faixa etária até 25 anos e na amostra total as correlações foram positivas e estatisticamente significativas entre as duas medidas com o TEADI. Em relação à magnitude dessas correlações, em ambos 
os casos foi baixa. Quanto às outras faixas etárias, as correlações não foram significativas. No caso dos 26 aos 35 anos, a magnitude da correlação foi nula, enquanto que na faixa etária de 36 anos ou mais a magnitude foi baixa entre o TEADI e a medida de Concentração e moderada com a medida de Velocidade com Qualidade. Em que pese a não terem sido significativas, o resultado não pode ser ignorado, uma vez que o número de sujeitos em ambas as faixas, principalmente na faixa de idade mais avançada, foi muito pequeno $(\mathrm{N}=5)$.

Ainda foi realizada uma análise com a medida de Sustentação do AS, sendo realizada uma ANOVA tendo como variável dependente a pontuação no TEADI. Os resultados não evidenciaram diferenças estatisticamente significativas entre os três grupos $[F(2,101)=2,36, p=0,100]$. Corroborando a ausência de diferenças, a prova de Tukey não diferenciou nenhum dos três grupos formados em função da Sustentação da atenção.

A continuação foi realizada uma correlação parcial, controlando o efeito da variável idade, tendo em vista que os instrumentos estudados nesta pesquisa afirmam a existência de uma relação inversa entre idade avançada e capacidade atencional. Os resultados dessas análises encontram-se na Tabela 2.

Tabela 2.

Coeficientes de Correlação Parciais entre o TEADI e as medidas de Concentração e de Velocidade com Qualidade do AD e AS, no total e por faixa etária.

\begin{tabular}{|c|c|c|c|c|c|}
\hline & & \multicolumn{4}{|c|}{$A D$} \\
\hline & & \multicolumn{2}{|c|}{ Concentração } & \multicolumn{2}{|c|}{$\begin{array}{c}\text { Velocidade com } \\
\text { Qualidade }\end{array}$} \\
\hline & & $r$ & $p$ & $r$ & $p$ \\
\hline \multirow[t]{7}{*}{ TEADI } & Até 25 anos & 0,75 & $<0,001$ & 0,65 & $<0,001$ \\
\hline & 26 a 35 anos & 0,78 & $<0,001$ & 0,45 & 0,046 \\
\hline & 36 anos ou & 0,55 & 0,031 & 0,53 & 0,033 \\
\hline & Amostra total & 0,72 & $<0,001$ & 0,56 & $<0,001$ \\
\hline & & \multicolumn{4}{|c|}{ AS } \\
\hline & & \multicolumn{2}{|c|}{ Concentração } & \multicolumn{2}{|c|}{$\begin{array}{c}\text { Velocidade com } \\
\text { Qualidade }\end{array}$} \\
\hline & & $r$ & $p$ & $r$ & $p$ \\
\hline \multirow[t]{4}{*}{ TEADI } & Até 25 anos & 0,26 & 0,036 & 0,29 & 0,016 \\
\hline & 26 a 35 anos & 0,09 & 0,653 & 0,20 & 0,331 \\
\hline & 36 anos ou & $-0,06$ & 0,945 & $-0,03$ & 0,967 \\
\hline & Amostra total & 0,22 & 0,024 & 0,28 & 0,005 \\
\hline
\end{tabular}


No caso da relação entre o TEADI e o AD, quando controlado o efeito da idade foi verificado que os coeficientes e a tendência das correlações mantiveram-se muito semelhantes em todas as faixas etárias e na amostra total. Dessa forma, evidencia-se que a relação entre as medidas de Concentração e de Velocidade com Qualidade do AD com o TEADI existe de fato, independentemente da idade.

Já nas medidas de Concentração e de Velocidade com Qualidade do AS com o TEADI, também foi observado que a tendência e a magnitude das correlações manteve-se estável, exceção feita à faixa etária de 36 anos ou mais, que apresentaram coeficientes de correlação negativos e ainda não significativos. Isso pode ser atribuído, talvez, ao número muito pequeno de sujeitos.

\section{DISCUSSÃO}

Em estudos psicométricos considera-se a correlação entre um novo teste com outros já existentes como evidência de validade de construto por permitir a avaliação do quanto eles mensuram uma mesma área de comportamento. Nesse procedimento, na hipótese de que os testes mensurem o mesmo construto esperam-se correlações moderadamente altas, medianas para construtos semelhantes, mas não idênticos, e nula para construtos diferentes, indicando a dissociação entre eles (Arías, 1996; Anastasi \& Urbina, 2000; Pasquali, 2001). Dentro dessa perspectiva, o objetivo deste estudo foi a busca de evidências de validade para o TEADI (Rueda, 2010) pela associação com o AD e o AS (Sisto et al, 2006). Em ambos os casos, a pontuação do TEADI foi correlacionada com os índices de Concentração e Velocidade com Qualidade do AD e do AS.

A correlação entre o TEADI e a medida de Concentração do AD apresentou predominantemente índices de correlação de magnitude alta, com exceção para a faixa etária a partir de 36 anos, para a qual foi moderada. No caso da associação com a medida de Velocidade com Qualidade as magnitudes foram mais baixas, sendo alta para o grupo até 25 anos, e moderada para os demais grupos etários e a amostra total. Em todos os casos obteve-se significância estatística a 5 ou 1\%. Quando controlado o efeito da idade sobre a correlação não houve mudanças importantes nem na magnitude das correlações nem no nível de significância, evidenciando estabilidade na correlação entre os dois testes independentemente do efeito da idade indicado pelos estudos de validade desenvolvimental (Rueda, 2009; Sisto et al, 2006). 
Em estudos semelhantes, ou seja, entre testes que avaliavam o mesmo tipo de atenção, observaram-se resultados similares. O AC, por exemplo, encontrou correlações variando de moderadas a muito altas, em sua maioria acima de 0,50, quando associado com outros testes de atenção concentrada, entre eles o TEACO-FF, o Teste de Atenção Concentrada de Toulouse-Piéron, o TACOM-A, o TACOM-B e o Teste D2 (Cambraia, 2003; Rueda, 2009). Esse padrão mantevese, apesar de pequena redução, quando controlado o efeito da idade (Rueda, 2009).

Assim, pode-se inferir que porcentagem importante do que o TEADI e o AD avaliam é composta de elementos comuns. Quanto à porcentagem que não é comum, pode-se pensar na possibilidade de os testes serem estruturalmente bastante diferentes. Nesse sentido, o AD é um teste impresso em cores no qual a pessoa deve procurar uma figura oval verde clara e duas figuras verde escuro lado a lado na horizontal ou na vertical, assim como também uma figura oval amarela e duas figuras laranjas lado a lado na horizontal ou na vertical. Por sua vez, no TEADI a pessoa deve procurar três estímulos isolados na cor preta. Ou seja, no caso do AD a divisão da atenção pode ser entendida como sendo intrateste (procurar dois ou mais estímulos dentre vários distratores) e intra-estímulo (procurar a combinação de figuras e cores dentro do mesmo estímulo), enquanto que no TEADI a procura pelos estímulos é apenas intra-teste.

Em oposição aos resultados acima discutidos, as correlações entre o TEADI e o AS apresentaram índices de correlação menores. De fato, houve predominância de magnitudes baixas, excetuando para a faixa etária entre 26 e 35 anos, que foi nula para a medida de Concentração e a partir de 36 anos que foi moderada. De modo geral, quando foi controlado o efeito da idade mantiveram-se os valores anteriormente descritos, com exceção para o grupo a partir de 36 anos, cujas magnitudes diminuíram passando de baixa e moderada, respectivamente com a medida de Concentração e Velocidade com Qualidade, para nulas. Assim como ocorreu nas análises entre o TEADI e o $A D, a$ manutenção das magnitudes de correlação entre o TEADI e o AS quando controlado o efeito da idade poderia indicar certa estabilidade na associação entre esses testes independentemente da variável idade, cuja influência foi determinada por estudos de validade desenvolvimental (Rueda, 2009; Sisto et al, 2006). 
Resultados semelhantes foram encontrados por Noronha et al (2006) quando associaram o AS ao AC, ou seja, correlações baixas e moderadas e redução nas magnitudes quando foi controlado o efeito da idade passando à baixa. Ao lado disso, quando o $A C$ foi relacionado ao AD as magnitudes também foram baixas e moderadas, mantendo-se quando controlado o efeito da idade (Sisto et al, 2006). Por sua vez, o TEACO-FF obteve índices moderados e positivos com o TTC (Rabelo et al, 2009) e com o AS tanto por sexo, quanto na amostra total, além de correlações nulas e baixas com o $A D$, predominantemente (Rueda, 2009). Do mesmo modo, Castro et al (2009) obtiveram correlações moderadas entre o MPM e o Teste de Atenção Seletiva Visual.

Todos esses estudos permitem uma mesma inferência, ou seja, a presença de uma comunalidade moderadamente baixa entre os testes indicando construtos semelhantes, mas não idênticos. Tais resultados suportam a hipótese aventada para a associação entre o TEADI e o AS, uma vez que ambos avaliam atenção, mas de tipos diferentes operacionalizados de forma distinta (Baptista et al, 2007; Benczik \& Casella, 2007; Gaddes \& Edgell, 1994; Sisto et al, 2006; Sternberg, 2000).

Um aspecto importante deste estudo foi a possibilidade de associação do TEADI com testes que avaliam dois tipos de atenção, sendo, um deles semelhante (AD) e outro diferente (AS) daquele avaliado pelo TEADI. Esse desenho metodológico permitiu avaliar em uma mesma amostra resultados que conceitualmente deveriam ser diferentes, o que de fato ocorreu.

Os participantes, embora apresentando uma boa variabilidade, tiveram uma média de idade baixa, indicando concentração de jovens abaixo dos 30 anos. Foi justamente no grupo a partir de 36 anos que surgiu uma correlação destoante entre o TEADI e o AS, sendo uma correlação moderada com a medida Velocidade com Qualidade, enquanto as demais foram baixas ou nulas. Foi também nesse grupo etário, e na correlação com o AS, que ocorreu a maior alteração quando controlado o efeito da idade. Essa correlação pode ser o reflexo do baixo número de participantes mais velhos, o que poderia ser verificado em outros estudos, principalmente, ao se observar que, de modo geral, a idade interferiu muito pouco nas magnitudes encontradas, tanto na associação com o AS quanto com o AD.

Com base nesses resultados podem-se estabelecer algumas conclusões. Inicialmente o TEADI obteve correlações mais altas com o AD, em comparação 
àquelas obtidas com o AS, o que era esperado. Dado os índices encontrados, isso indica que o TEADI e o AD avaliam o mesmo construto, no caso, a atenção dividida, mas com algumas diferenças provavelmente relacionadas à sua operacionalização. Em contraposição, no segundo caso, observa-se que embora o TEADI e o AS possuam elementos comuns, não avaliam o mesmo construto, mas construtos semelhantes, ou seja, dois tipos de atenção. Assim, conclui-se pela evidência de validade de construto para o TEADI pela associação com o AD e o AS.

\section{REFERÊNCIAS}

Anastasi, A., \& Urbina, S. (2000). Testagem psicológica. Porto Alegre: Artmed. Arías, R. M. (2006). Psicometría: Teoria de los tests psicológicos y educativos. Madrid: Síntesis Psicología.

Baptista, M. N., Rueda, F. J. M., \& Sisto, F. F. (2007). Relação entre estresse laboral e atenção concentrada. Encontro: Revista de Psicologia, XI(16), 7589.

Braga, J. L. (2007). Atenção concentrada e atenção difusa: Instrumentos de medida. Dissertação de Mestrado, Faculdade de Psicologia, Universidade de Brasília, Brasília.

Cambraia, S. V. (2003). Teste de Atenção Concentrada (AC). Manual. São Paulo: Vetor Editora Psicopedagógica Ltda.

Casella, E. B. (2007). Atenção. In P. W. Schelini. (Org.). Alguns domínios da avaliação psicológica. (pp. 31-58). Alínea Editora: Campinas.

Castro, N. R. (2009). Construção de um teste de atenção seletiva visual. Dissertação de Mestrado, Faculdade de Psicologia, Universidade São Francisco, Itatiba.

Castro, N. R., Sisto, F. F., \& Silva, M. A. (2009). Evidências de validade para o Teste de Atenção Seletiva Visual. In Anais do IV Congresso Brasileiro de Avaliação Psicológica/XIV Conferência Internacional de Avaliação Psicológica: formas e contextos, Campinas, SP: Instituto Brasileiro de Avaliação Psicológica.

Gaddes, W. H., \& Edgell, D. (1994). Learning disabilities and brain function: A neuropsychological approach. New York: Springer-Verlag.

Gazzaniga, M. S., Ivry, R. B., \& Mangun, G. R. (2006). Neurociência cognitiva: A biologia da mente. Porto Alegre: Artmed. 
Lima, F. R. (2005). Compreendendo os mecanismos atencionais. Ciência \& Cognição, 6, 113-122. Retirado em: 15/03/2006, de Ciência \& Cognição, www.cienciaecognicao.org.br.

Mira, A. M. G. (2004). PMK: Psicodiagnóstico Miocinético. São Paulo: Vetor

Noronha, A. P. P., Sisto, F. F., Bartholomeu, D., \& Rueda, F. J. M. (2006). Atenção sustentada e concentrada: Construtos semelhantes? Psicologia: Pesquisa \& Trânsito, 2(1), 29-36.

Pasquali, L. (2001). Parâmetros psicométricos dos testes psicológicos. In L. Pasquali. (Org.). Técnicas de Exame Psicológico - TEP. (pp. 111-136). São Paulo: Casa do Psicólogo.

Rabelo, I. S., Pacanaro, S. V., Castro, N. R., Leme, I. A. S., Rossetti, M. O., \& Güntert, C. M. (2009). Evidências de validade do Teste das Trilhas Coloridas por meio do Teste de Atenção Concentrada (TEACO-FF). In Anais do IV Congresso Brasileiro de Avaliação Psicológica/XIV Conferência Internacional de Avaliação Psicológica: formas e contextos, Campinas, SP: Instituto Brasileiro de Avaliação Psicológica.

Rueda, F. J. M. (2009). Evidência de validade para o Teste de Atenção Concentrada - TEACO-FF. Tese de Doutorado. Universidade São Francisco.

Rueda, F. J. M. (2010). Teste de Atenção Dividida (TEADI): Manual técnico. Itatiba: Universidade São Francisco.

Rueda, F. J. M., \& Sisto, F. F. (2009). Teste de Atenção Concentrada (TEACOFF). Manual. São Paulo: Casa do Psicólogo.

Sisto, F. F. (2007). Delineamento correlacional. In M. N. Baptista \& D. C. de Campos (Orgs). Metodologias de pesquisa em ciências: análises quantitativa e qualitativa. (pp. 90-101). Rio de Janeiro: LTC editora.

Sisto, F. F., Noronha, A. P. P., Lamounier, R., Bartholomeu, D., \& Rueda, F. J. M. (2006). Testes de Atenção Dividida e Sustentada (AD e AS). Manual. São Paulo: Vetor Editora Psicopedagógica Ltda.

Sternberg, R. J. (2000). Psicologia cognitiva. Porto Alegre: Artmed.

Contato: fabianrueda@saofrancisco.edu.br

Recebido em: 04/10/2010

Revisado em: 17/11/2010

Aceito em: 01/12/2010 